\title{
Patchy and Janus Nanoparticles by Self- Organization of Mixtures of Fluorinated and Hydrogenated Alkanethiolates on the Surface of a Gold Core
}

\author{
Maria Şologan, ${ }^{\dagger}$ Domenico Marson, ${ }^{\ddagger}$ Stefano Polizzi, ${ }^{\S}$ Paolo Pengo, ${ }^{\dagger}$ Silvia Boccardo, ${ }^{\ddagger}$ Sabrina Pricl, ${ }^{\ddagger}$ \\ Paola Posocco, $*,+, \perp$ and Lucia Pasquato $*,+, \perp$ \\ ${ }^{\dagger}$ Department of Chemical and Pharmaceutical Sciences and INSTM Trieste Unit, via L. Giorgieri 1, and ${ }^{\ddagger}$ MOSE Laboratory, \\ Department of Engineering and Architecture and INSTM Trieste Unit, University of Trieste, Piazzale Europa 1, 34127 Trieste, Italy \\ ${ }^{\S}$ Department of Molecular Sciences and Nanosystems, University Ca' Foscari of Venezia, via Torino 155/b, I-30172 Venezia-Mestre, \\ Italy
}

Supporting Information

ABSTRACT: The spontaneous self-organization of dissimilar ligands on the surface of metal nanoparticles is a very appealing approach to obtain anisotropic "spherical" systems. In addition to differences in ligand length and end groups, a further thermodynamic driving force to control the self-assembled monolayer organization may become available if the ligands are inherently immiscible, as is the case of hydrogenated (H-) and fluorinated (F-) species. Here, we validate the viability of this approach by combining ${ }^{19} \mathrm{~F}$ NMR experiments and multiscale molecular simulations on large sets of mixed-monolayer-protected gold nanoparticles (NPs). The

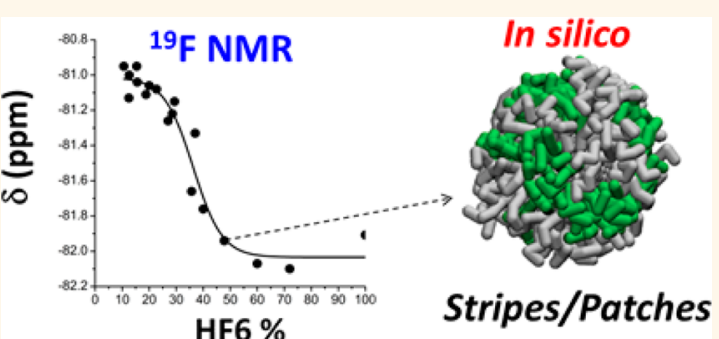
phase segregation of blends of F- and H-thiolates grafted on the surface of gold NPs allows a straightforward approach to patterned mixed monolayers, with the shapes of the monolayer domains being encoded in the structure of the $\mathrm{F} / \mathrm{H}$-thiolate ligands. The results obtained from this comprehensive study offer molecular design rules to achieve a precise control of inorganic nanoparticles protected by specifically patterned monolayers.

KEYWORDS: anisotropic nanoparticles, phase segregation, self-assembly, gold nanoparticles, multiscale molecular modeling, NMR

$\mathrm{P}$ atchy particles display precisely controlled surface areas with different properties. These different surface areas determine particle-environment interactions, as nicely demonstrated by several examples of naturally occurring anisotropic particles such as pollen grains ${ }^{1}$ or viruses. ${ }^{2}$ Surface morphology is also responsible for the biological activity of these systems; thus, for instance, Janus-like structures of hydrophobin proteins can have roles in the attachment of fungi to different surfaces. $^{3}$ The development of artificial (nano)particles with anisotropy in shape, surface properties, and chemical functionality will certainly lead to innovative materials. ${ }^{4-7}$ Indeed, taking inspiration from Nature, chemists now utilize patchy particles as elemental building blocks for the generation of new materials, mainly by exploiting their self-assembly properties in the design of $2 \mathrm{D}$ or $3 \mathrm{D}$ networks. By this approach, hierarchical structures with applications in biology/medicine and in the mimicry of viruses have been obtained. ${ }^{8-11}$ This would have been difficult or even out of reach using colloids displaying solely isotropic interactions. $^{12}$
While many studies carried out in this field concern polymeric particles with size greater than $100 \mathrm{~nm}$, hybrid organic-inorganic nanoparticles characterized by dimensions less than $100 \mathrm{~nm}$ have been much less explored.

In this context, the preparation of spherical metal nanoparticles decorated by anisotropic organic monolayers remains challenging, notwithstanding the easy and precise control of their size, surface functionalization, and dispersion. Also, because of their inorganic core, these systems may be endowed with interesting additional properties with respect to soft (e.g., polymeric) particles. In this direction, tremendous efforts have been devoted to theoretical and experimental studies aimed at inducing the organization of thiolate ligands on the surface of gold nanoparticles (NPs) into well-defined domains as a source of anisotropy. Indeed, several different approaches for the

Received: June 14, 2016

Accepted: September 23, 2016

Published: September 23, 2016 

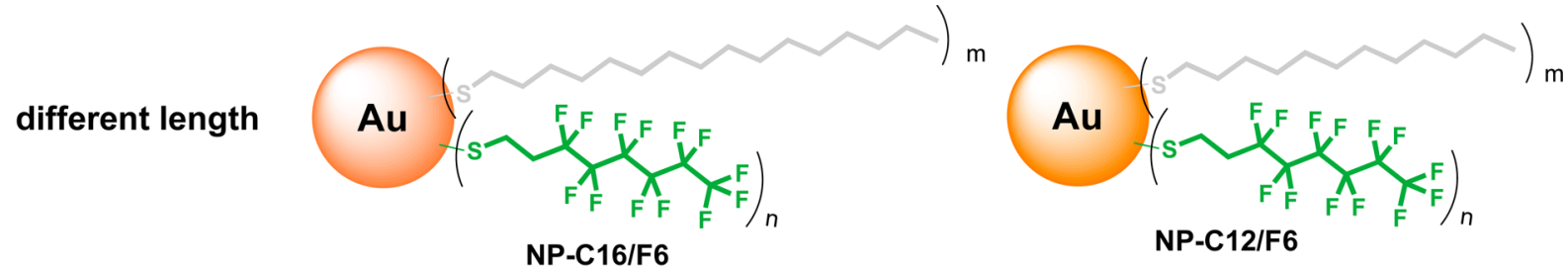

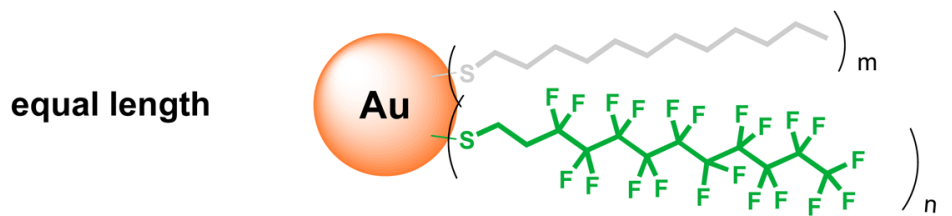

NP-C12/F10

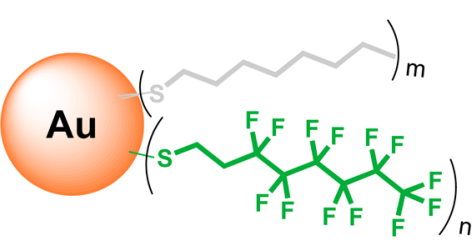

NP-C8/F6

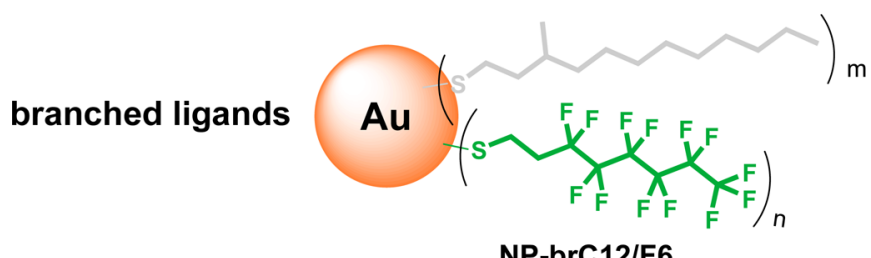

Figure 1. Representation of the library of mixed-monolayer-protected gold NPs investigated in this work.

preparation of patchy or Janus hybrid organic-inorganic NPs have been reported. ${ }^{13}$ Particularly attractive in this respect is the formation of anisotropic spherical nanoparticles by exploiting the spontaneous self-assembly of coded ligands on their surface. This strategy was pioneered by the group of Stellacci, using mixtures of immiscible thiolate ligands that self-sort on the gold NP surface forming stripe-like domains. ${ }^{14,15}$ These experimental results were coupled to computational investigations carried out by Glotzer and co-workers, who identified the difference in ligand length and the NP core size as two critical parameters in determining the morphology of self-assembled monolayers (SAMs). ${ }^{16}$ Thus, according to these seminal studies, binary mixtures of thermodynamically incompatible thiolates can originate three main SAM morphologies on a gold NP surface, depending on their structure and on NP core size: Janus NPs are foreseen when the ligands have comparable length or the NP core diameter is small (typically below $2.5 \mathrm{~nm}$ ); ${ }^{17}$ patches or stripe-like domains are predicted when the two ligands differ in size, while a random organization of the monolayer is expected when one of the two ligands has a branched structure. ${ }^{18}$ According to this rationale, several thiol combinations, such as mercaptopropionic acid and octane- or dodecanethiol, ${ }^{14,15}$ mercaptopropanesulfonate and phenylmethanethiol, ${ }^{19}$ thiopro$\mathrm{nin}$ and mercaptoundecyltetraethylene glycol, ${ }^{20} 11$-mercapto-1undecanesulfonate and octanethiol, ${ }^{18}$ octanethiol and $N-1-\{2-[2-$ (2-methoxyethoxy)ethoxy] ethyl $\}$-8-sulfanyloctanamide (HSC8-TEG), ${ }^{21}$ and dodecanethiol and diphenyl thiol, ${ }^{22}$ have been experimentally explored.

In this respect, we reasoned that combining $\mathrm{H}$ - and $\mathrm{F}$ alkanethiolates in the NP decorating monolayer should allow the ligand immiscibility (Flory-Huggins $\chi$ parameter) to be maximized and self-sorting to be promoted, in analogy to a $2 \mathrm{D}$ SAM. $^{23}$

In addition, the presence of fluorinated ligands in the coating of hybrid organic-inorganic nanoparticles is of particular interest in material science because of their electronic properties, which may influence the features of the metal core. ${ }^{24-26}$ Moreover, their antifouling surface properties may be exploited at the interface with complex fluids or, even more importantly, in their interactions with biological tissues. So far, these features are unexplored for monolayer-protected metal nanoparticles due to limited examples of fluorinated nanoparticles that are soluble in organic or aqueous solvents.

In this context, we reported examples of 3D mixed monolayers composed of $\mathrm{F}$ - and $\mathrm{H}$-thiolates ending with a polyethylene glycol (PEG) group ${ }^{27}$ and demonstrated that phase segregation occurs at all molar fractions explored; moreover, we found that the difference in ligand chain length determines the formation of ribbon-like domains on nanoparticles with a gold core larger than $2 \mathrm{~nm} .{ }^{28}$ Yet, the presence of a PEG terminal group did not allow for a complete dissection of the contribution of the immiscible hydrocarbon/fluorocarbon chains to the morphology of the monolayer.

To unravel this contribution and to further analyze the ligand structural requirements needed to induce well-defined morphologies, we embarked in the synthesis and systematic morphological investigation of gold nanoparticles protected by blends of $\mathrm{F}$ - and $\mathrm{H}$-alkanethiolates differing in chain length, steric bulk, and relative ratio. This study also allows assessing how the morphology correlates with NP properties such as solubility or aggregation.

Thus, we prepared 2-4 $\mathrm{nm}$ core diameter gold NPs decorated by blends (Figure 1) of F- and H-ligands of (i) different length: NP-C16/F6, protected by blends of hexadecanethiol (HC16) and $1 H, 1 H, 2 H, 2 H$-perfluorooctanethiol (HF6), and NP-C12/ F6, coated by mixtures of dodecanethiol (HC12) and HF6; (ii) equal length: NP-C12/F10, coated by $\mathbf{H C 1 2}$ and $1 H, 1 H, 2 H, 2 H$ perfluorododecanethiol (HF10), and NP-C8/F6, protected by mixtures of octanethiol (HC8) and HF6; and (iii) increased steric hindrance: NP-brC12/F6, featuring mixtures of 3-methyldodecane-1-thiol (HbrC12) and HF6. The complete list of the systems investigated in this work is reported in Tables S1-S5, and the synthetic strategies for the preparation of these nanoparticles have been recently described by some of us. ${ }^{29}$ 

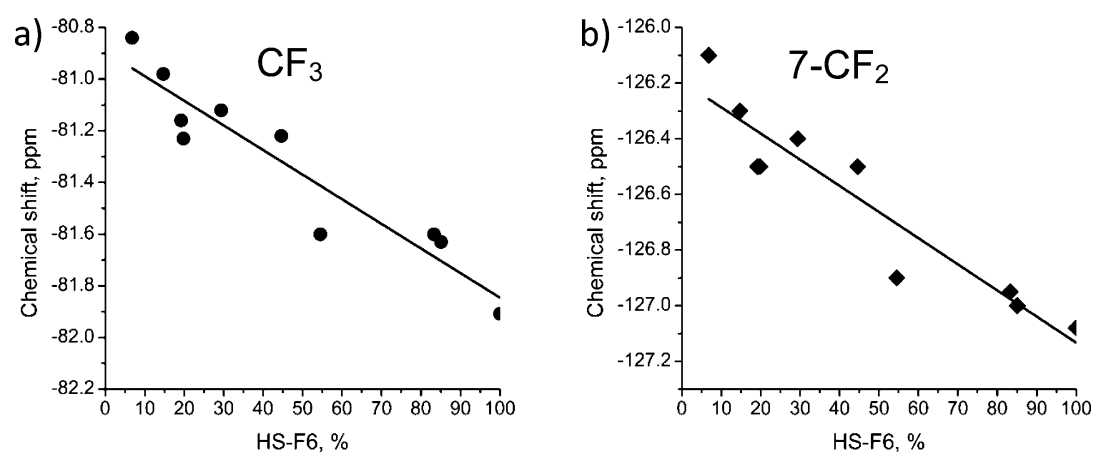

Figure 2. Chemical shift $(\delta)$ variation of $(a) \mathrm{CF}_{3}$ groups and $(\mathrm{b}) 7-\mathrm{CF}_{2}$ nuclei increasing the percentage of the fluorinated ligand in the monolayer of NP-brC12/F6. Solid line serves as a guide for the eyes only.

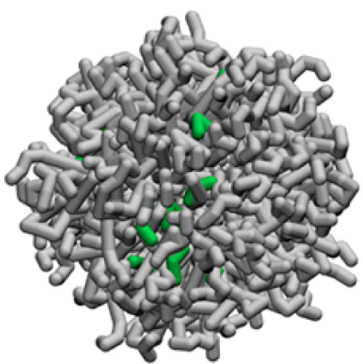

NP-brC12/F6-a

$\mathrm{Au}_{2230} \mathrm{brC} 12_{305} \mathrm{~F}_{22}$ $6.8 \%$ of $\mathrm{F} 6$

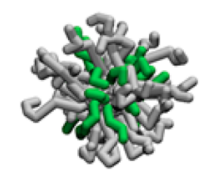

NP-brC12/F6-f

$\mathrm{Au}_{208} \mathrm{brC1}{ }_{30} \mathrm{~F}_{24}$ $44.6 \%$ of $\mathbf{F 6}$

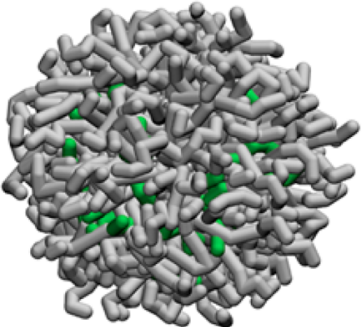

NP-brC12/F6-b

$\mathrm{Au}_{1830} \mathrm{brC} 12_{250} \mathrm{F6}_{43}$ $14.7 \%$ of $F 6$

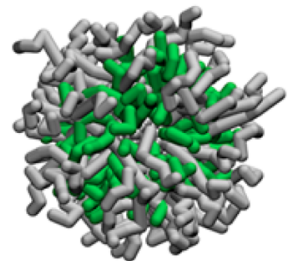

NP-brC12/F6-g

$\mathrm{Au}_{807} \mathrm{brC} 12_{76} \mathrm{~F}_{92}$ $54.5 \%$ of $\mathbf{F 6}$

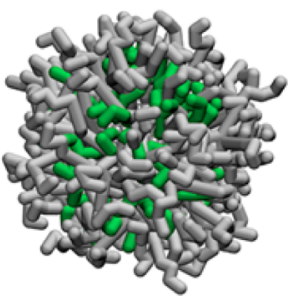

NP-brC12/F6-e

$\mathrm{Au}_{976} \mathrm{brC}_{12}{ }_{148} \mathrm{~F} 6_{62}$ $29.4 \%$ of $\mathbf{F} 6$

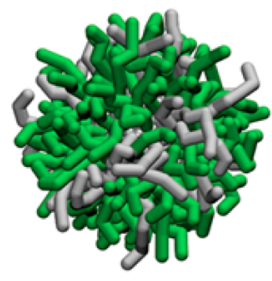

NP-brC12/F6-h

$\mathrm{Au}_{500} \mathrm{brC} 12_{28} \mathrm{~F} 6_{139}$ $83.3 \%$ of $\mathbf{F 6}$

Figure 3. Equilibrium morphologies of NP-brC12/F6 (color code: gray, brC12; green, F6) NPs as obtained by the mesoscale simulations. Solvent is omitted for clarity.

The assessment of the morphology of resulting mixedmonolayer-protected gold NPs may be cumbersome because of a large number of limitations such as their low solubility in common organic solvents, presence of impurities, or the slippery nature of the NP surface, thereby restricting the number of techniques suitable to study their morphology. To overcome some of these practical obstacles, in this work, we adopted an integrated and complementary experimental/computational approach for the characterization of structural features of these mixed SAM-protected gold NPs. Specifically, our experimental analysis relies on $1 \mathrm{D}$ and $2 \mathrm{D}{ }^{19} \mathrm{~F}$ NMR. NMR techniques based on ${ }^{1} \mathrm{H}$ NMR experiments have been successfully used in previous works to characterize monolayer-protected gold nanoparticles ${ }^{30}$ and, in particular, to investigate the morphology of mixed monolayers by complexation of lanthanide ions ${ }^{21}$ or by NOESY experiments. ${ }^{31}$ More recently, an NMR-based approach has been proposed to detail the shell morphology of NPs coated with binary mixtures of aliphatic and aromatic ligands exploiting a combination of chemical shift variations and NOESY experiments. ${ }^{22}$ Yet, the ${ }^{19} \mathrm{~F}$ NMR technique is definitely more sensitive to the chemical environment than ${ }^{1} \mathrm{H}$ NMR since ${ }^{19} \mathrm{~F}$ chemical shifts span more than $200 \mathrm{ppm}$, whereas proton chemical shift varies between 0 and $15 \mathrm{ppm}$. Accordingly, in this work, we exploited this technique further to sensitively probe changes in the environment surrounding the F-ligands as a function of monolayer composition. The theoretical investigation is based on a predictive multiscale molecular simulation protocol, that is, a combination of atomistic/coarse-grained calculations. ${ }^{28}$

\section{RESULTS AND DISCUSSION}

Gold nanoparticles NP-C16/F6, NP-C8/F6, and NP-brC12/ F6 were prepared by direct synthesis, whereas NP-C12/F6 and NP-C12/F10 were obtained by place exchange reaction from narrowly dispersed NP-C12, in turn prepared following the method of Miyake. ${ }^{32}$ Synthetic details are reported in ref 29 and, for additional nanoparticles, in the Supporting Information (SI). All samples were fully characterized using UV-vis, transmission electron microscopy (TEM), thermogravimetric analysis, and standard NMR experiments. NP solubility was also assessed and analyzed by increasing the molar fraction of the fluorinated ligands. ${ }^{29}$ This property was ultimately rationalized in light of the morphology of the 3D SAM. Monodimensional ${ }^{19} \mathrm{~F}$ NMR experiments were used to monitor the change in chemical shift as 

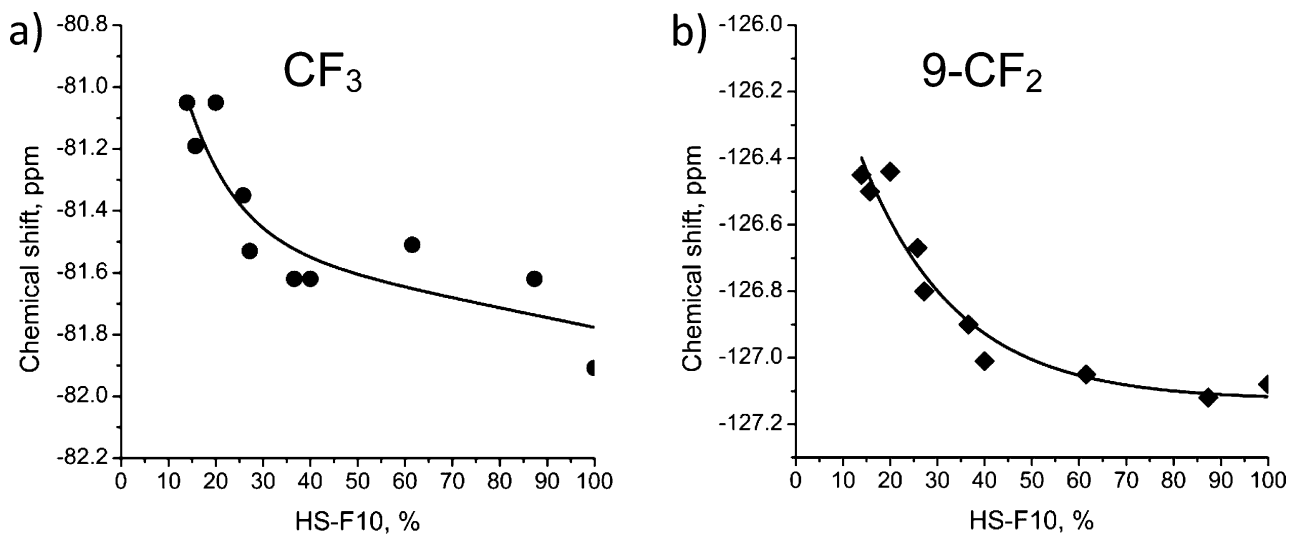

Figure 4. Chemical shift $(\delta)$ variation of $(a) C F_{3}$ groups and $(b) 9-C_{2}$ nuclei increasing the percentage of the fluorinated ligand in the monolayer of NP-C12/F10. Solid line serves as a guide for the eyes only.

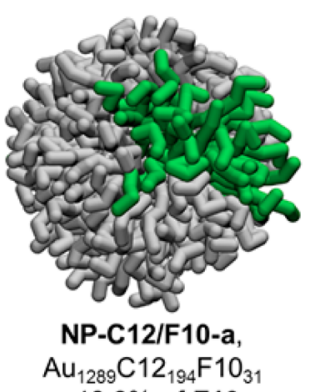

$13.9 \%$ of $\mathbf{F} 10$

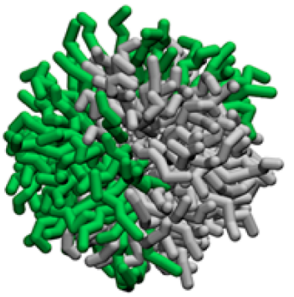

NP-C12/F10-f

$\mathrm{Au}_{1289} \mathrm{C} 12_{136} \mathrm{~F}_{10} 0_{78}$ $36.6 \%$ of $\mathbf{F 1 0}$
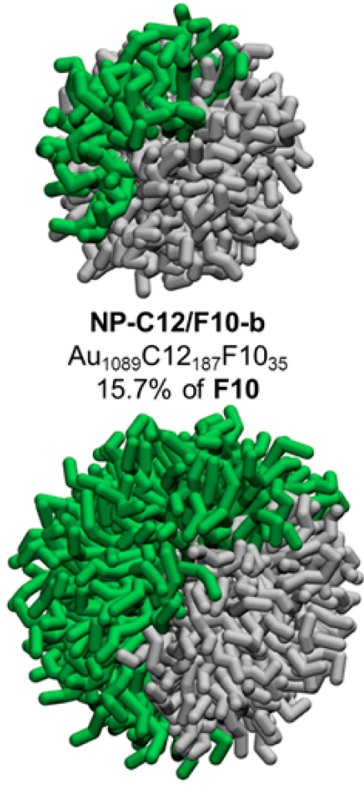

NP-C12/F10-h

$\mathrm{Au}_{2406} \mathrm{C} 12_{126} \mathrm{~F} 10_{200}$ $61.5 \%$ of $\mathbf{F 1 0}$

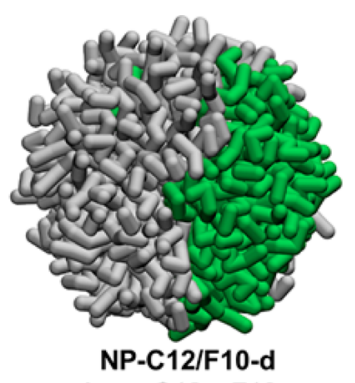

$\mathrm{Au}_{1830} \mathrm{C} 12_{211} \mathrm{~F} 10_{73}$ $25.8 \%$ of $\mathbf{F} 10$

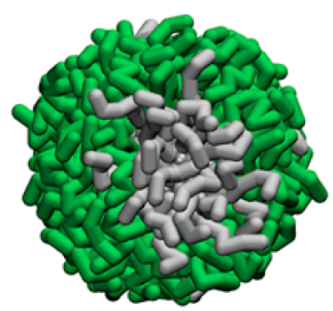

NP-C12/F10-i

$\mathrm{Au}_{1289} \mathrm{C} 12_{27} \mathrm{~F}_{10} 0_{186}$

$87.3 \%$ of $\mathbf{F 1 0}$

Figure 5. Equilibrium morphologies of NP-C12/F10 (color code: gray, C12; green, F10) obtained by the mesoscale simulations. Solvent is omitted for clarity.

a function of the percentage of the fluorinated thiolate in the monolayer. Due to solubility limitations, those nanoparticles soluble only in $\mathrm{CHCl}_{3}$ were dissolved in $\mathrm{CDCl}_{3}$ while the remaining samples were solubilized in different mixtures of $\mathrm{CDCl}_{3} / \mathrm{C}_{6} \mathrm{~F}_{6}$, as reported in Tables $\mathrm{S} 1-\mathrm{S} 5$. All ${ }^{19} \mathrm{~F}$ NMR spectra have been recorded using $\mathrm{CFCl}_{3}$ as an external reference. The ${ }^{19} \mathrm{~F}$ NMR spectra of the nanoparticles show only two peaks that are well-separated, allowing an accurate measurement of the chemical shift: the peak around $-81 \mathrm{ppm}$ represents the $\mathrm{CF}_{3}$ end group, and the peak at $-126 \mathrm{ppm}$ is assigned to the $\mathrm{CF}_{2}$ group next to the $\mathrm{CF}_{3}$ (Figure S2). The ${ }^{19} \mathrm{~F}$ NMR spectra indicate that the peaks of nanoparticles shift by changing the composition of the solvent. For this reason, the chemical shifts of the $\mathrm{CF}_{3}$ and of 7- or 9- $\mathrm{CF}_{2}$ groups have been corrected for all samples as described in SI for NP-C16/F6 as an example. Also, the ${ }^{19} \mathrm{~F}$ NMR data reveal that small changes of gold core size, within the same type of NPs, do not influence the chemical shift.
At the same time, multiscale molecular simulations have been carried out on the same set of NPs in order to predict the mixed monolayer organization. Coarse-grained methods as dissipative particle dynamics (DPD) are especially suitable for predicting self-assembling phenomena as those involved in the organization of binary mixtures of ligands on solid curved surfaces because they allow longer sampling times with respect to atomic resolution techniques such as molecular dynamics (MD). The computational approach applied here consists of exploiting information obtained at lower-level simulations (e.g., $\mathrm{MD}$ simulations) as input for higher-level calculations (e.g., mesoscopic DPD simulations) in a multiscale framework. DPD simulations were employed to study the effect of mixing different immiscible thiols on the surface of Au NPs in the presence of explicit solvent. With the aim of mimicking the experimental measurement conditions, both $\mathrm{CHCl}_{3}$ and $\mathrm{C}_{6} \mathrm{~F}_{6}$ were modeled in the relative appropriate amount (Tables $\mathrm{S} 1-\mathrm{S} 5$ ). In order to 

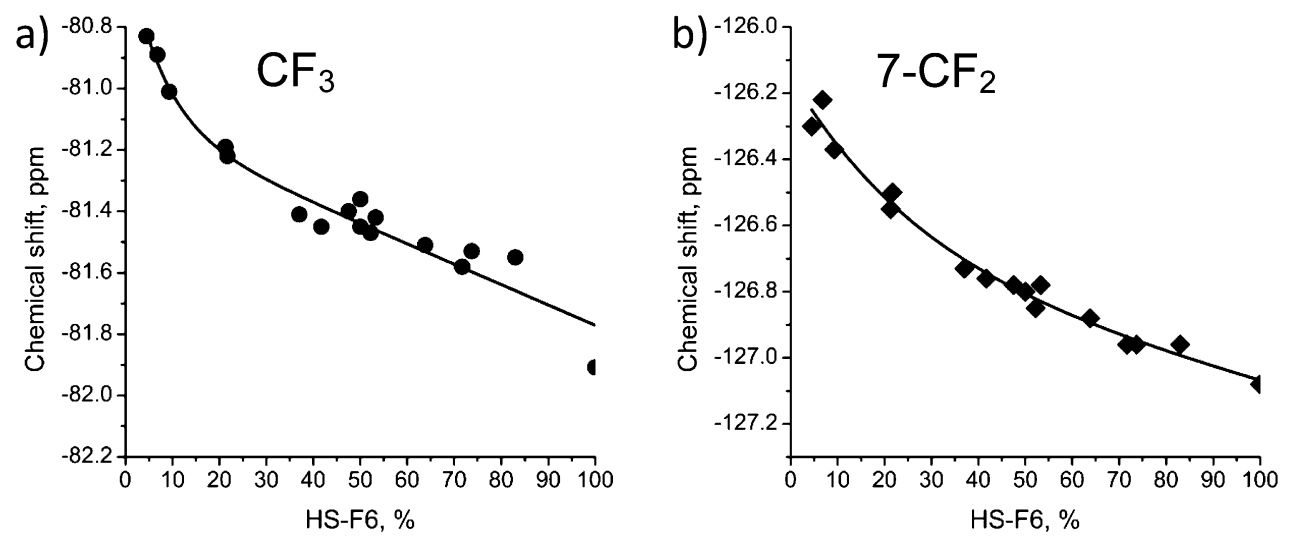

Figure 6. Chemical shift $(\delta)$ variation of $(a) \mathrm{CF}_{3}$ groups and $(\mathrm{b}) 7-\mathrm{CF}_{2}$ nuclei increasing the percentage of the fluorinated ligand in the monolayer of NP-C8/F6. Solid line serves as a guide for the eyes only.
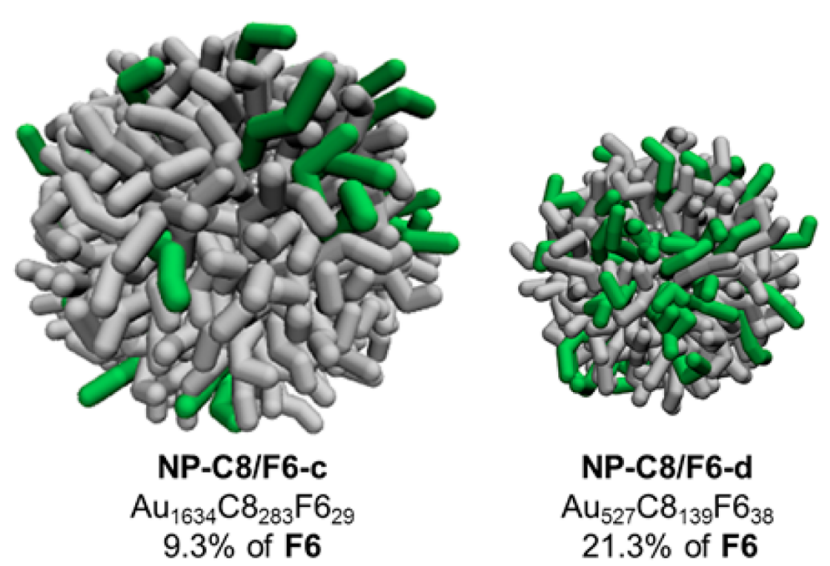

$\mathrm{Au}_{527} \mathrm{C}_{139} \mathrm{~F} 6_{38}$

$21.3 \%$ of $\mathbf{F 6}$

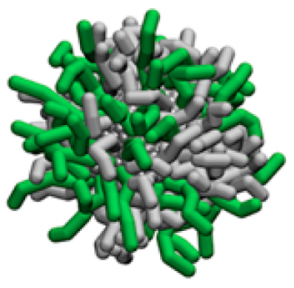

NP-C8/F6-g

$\mathrm{Au}_{475} \mathrm{C}_{75} \mathrm{F6}_{53}$

$41.7 \%$ of $\mathbf{F} 6$

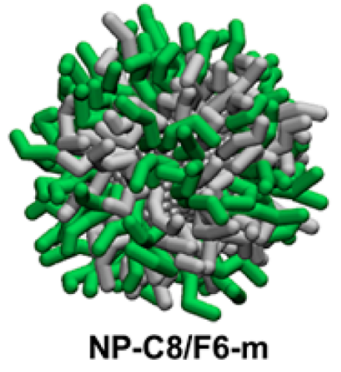

$\mathrm{Au}_{807} \mathrm{C}_{86} \mathrm{~F}_{98}$ $53.3 \%$ of $\mathrm{F} 6$

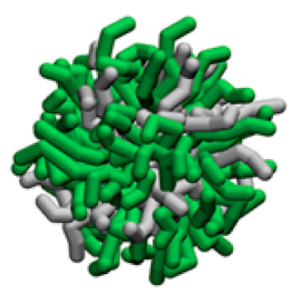

NP-C8/F6-O

$\mathrm{Au}_{459} \mathrm{C}_{32} \mathrm{F6}_{80}$

$71.7 \%$ of F6

Figure 7. Equilibrium morphologies of NP-C8/F6 (color code: gray, C8; green, F6) NPs as obtained by the mesoscale simulations. Solvent is omitted for clarity.

reproduce a realistic gold core, an icosahedral shape was adopted. Mesoscale models and procedure are described in detail in the SI.

Effect of Solvent Composition on the Chemical Shift.

From the experiments reported in Figures S4 and S5 for NPC16/F6, it can be observed that the solvent composition has a smaller influence on the chemical shift of the peak corresponding to $7-\mathrm{CF}_{2}$ groups with respect to that exerted on the $\mathrm{CF}_{3}$ groups. For this reason, we initially surmised that the variation of the chemical shift of $\mathrm{CF}_{3}$ would be larger than that of $\mathrm{CF}_{2}$ groups as a function of the loading of the fluorinated thiolates into the monolayer. On the contrary, even though the $\mathrm{CF}_{3}$ group is more exposed to the solvent than the $\mathrm{CF}_{2}$ group, the variation of their chemical shift as a function of the monolayer composition is similar in both cases. These results further confirm that the chemical shift variations reported here are not a consequence of the variation of the solvent composition but, instead, result from an evolution of the monolayer organization.

Nanoparticles Coated by Branched Ligands, NPbrC12/F6. The chemical shifts of the $\mathrm{CF}_{3}$ and $7-\mathrm{CF}_{2}$ groups of ligand F6 as a function of the percentage of the fluorinated ligand into the monolayer for the NP-brC12/F6 system are shown in Figure 2. These graphs display that the broad peaks of the nanoparticles gradually shift upfield when the percentage of F6 into the monolayer increases from 6 to $100 \%$. The linear decay of Figure 2 suggests that the average composition of the first nearest neighbor shell of each F-ligand coincides with the overall composition of the monolayer. This is in agreement with the initial hypothesis that the branched structure of the hydrogenated ligand does not allow an ordered crystalline arrangement on the surface of the gold core, hampering the 
a)

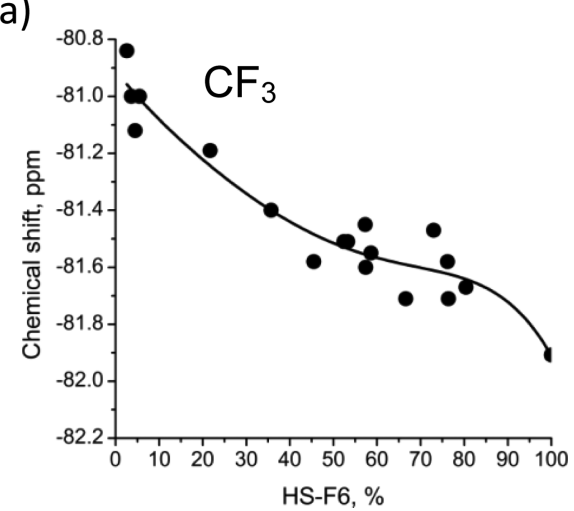

b)

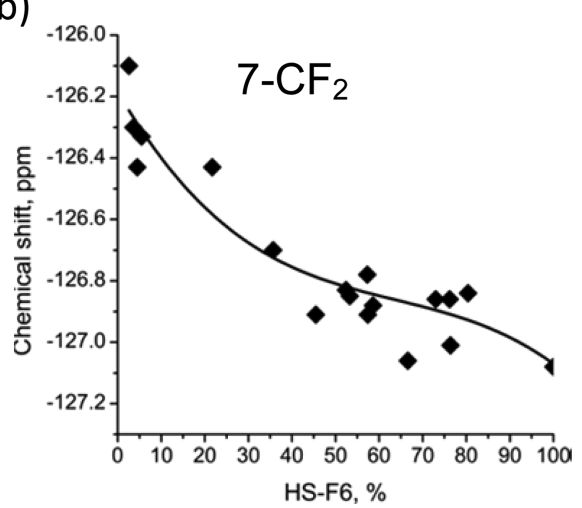

Figure 8. Chemical shift $(\delta)$ variation of $(a) \mathrm{CF}_{3}$ groups and $(\mathrm{b}) 7-\mathrm{CF}_{2}$ nuclei increasing the percentage of the fluorinated ligand in the monolayer of NP-C16/F6. Solid line serves as a guide for the eyes only.

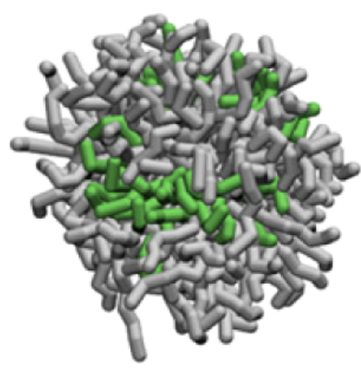

NP-C16/F6-e

$\mathrm{Au}_{976} \mathrm{C} 16_{147} \mathrm{Fb}_{42}$

$21.7 \%$ of $\mathbf{F} 6$

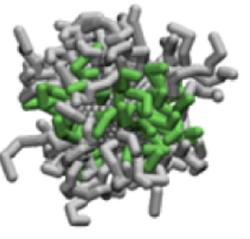

NP-C16/F6-g

$\mathrm{Au}_{309} \mathrm{C} 16_{42} \mathrm{F6}_{35}$

$45.5 \%$ of $\mathbf{F 6}$

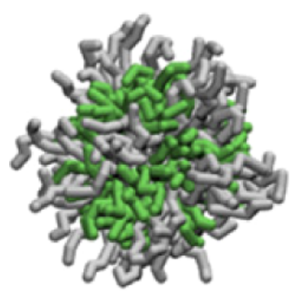

NP-C16/F6-j

$\mathrm{Au}_{475} \mathrm{C} 16_{71} \mathrm{F6}_{96}$

$57.3 \%$ of $\mathbf{F 6}$

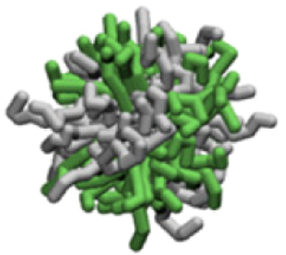

NP-C16/F6-n

$\mathrm{Au}_{314} \mathrm{C} 16_{23} \mathrm{F6}_{63}$

$73.0 \%$ of $\mathrm{F} 6$

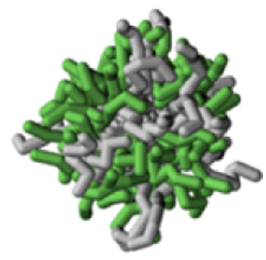

NP-C16/F6-q

$\mathrm{Au}_{314} \mathrm{C} 16_{18} \mathrm{~F}_{72}$

$80.4 \%$ F6

Figure 9. Equilibrium morphologies of selected of NP-C16/F6 (color code: gray, C16; green, F6) NPs as obtained by mesoscale simulations. Solvent is omitted for clarity.

formation of phase-segregated domains. This hypothesis is confirmed by the equilibrium morphologies collected from the corresponding DPD calculations summarized in Figure 3. These simulation structures clearly prove that the two ligands have no tendency to form compact domains, and they prefer to remain isolated; this results in a random organization of the monolayer regardless of the monolayer composition and dimension of the gold core. This evidence agrees well with previous literature data on the organization of monolayers comprising branched thiolates. $^{18}$

Nanoparticles Coated by Ligands of Equal Length, NPC12/F10 and NP-C8/F6. The behavior of the chemical shifts of the $\mathrm{CF}_{3}$ and $9-\mathrm{CF}_{2}$ groups versus the percentage of fluorinated ligands into the monolayer for samples of NP-C12/F10 (Figure 4) is different from that observed for NP-brC12/F6, and the interpolation of the points conforms to a $1 /\left(\right.$ F10\%) decay. ${ }^{22} \mathrm{~A}$ steep decay is observed when the loading of the fluorinated ligand is less than $40 \%$, indicating a strong evolution of the surface area at the $\mathrm{H} / \mathrm{F}$ interface; at higher percentages, the chemical shift remains nearly constant, suggesting no significant changes at the $\mathrm{H} / \mathrm{F}$ interface.

The equilibrium morphologies obtained from the mesoscale simulation of the NP-C12/F10 systems, presented in Figure 5, reveal that the ligands form two distinct domains; that is, the SAM has a Janus-type morphology, in line with a previously reported theoretical prediction on nanoparticles protected by ligands of equal length. ${ }^{16}$ Indeed, to our knowledge, there are no previous examples of 3D monolayers with Janus morphology obtained by place exchange in the absence of external tools. This organization of the monolayer explains the behavior of the chemical shift of the $\mathrm{CF}_{3}$ and $9-\mathrm{CF}_{2}$ groups as a function of the monolayer composition.

These results are also supported by the NPs' tendency to form dimers under TEM conditions, as recently reported for a nanoparticle featuring a Janus organization of the decorating shell. $^{29}$ 

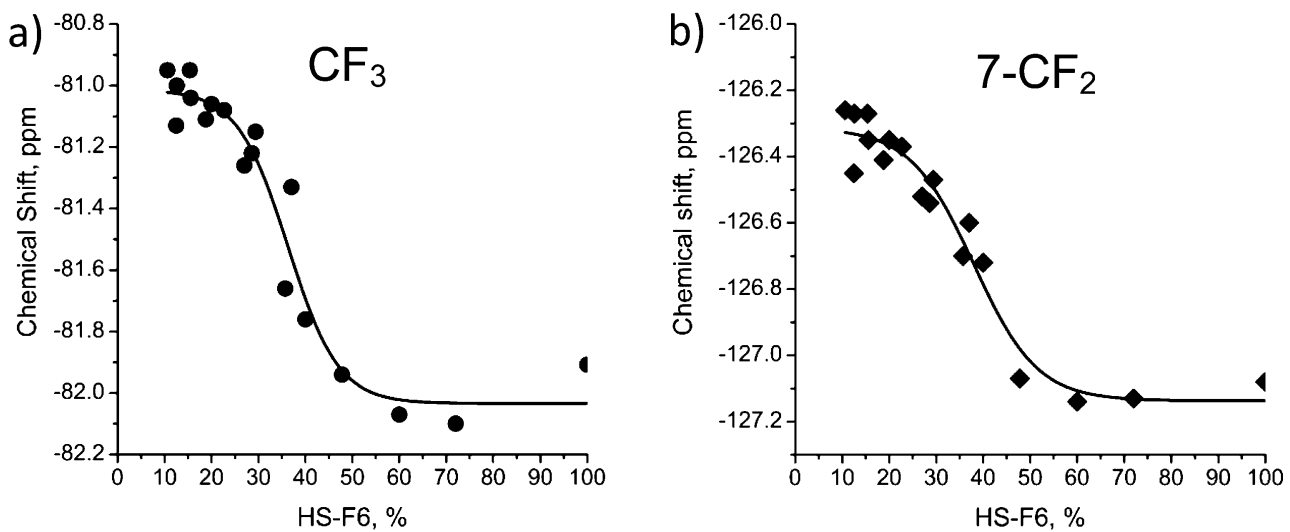

Figure 10. Chemical shift $(\delta)$ variation of $(a) C F_{3}$ groups and $(b) 7-C F_{2}$ nuclei increasing the percentage of the fluorinated ligand in the monolayer of NP-C12/F6. Solid line serves as a guide for the eyes only.

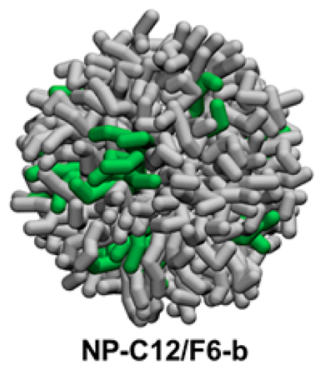

$\mathrm{Au}_{1310} \mathrm{C} 12_{210} \mathrm{~F} 6{ }_{30}$

$12.5 \%$ of $\mathbf{F} 6$

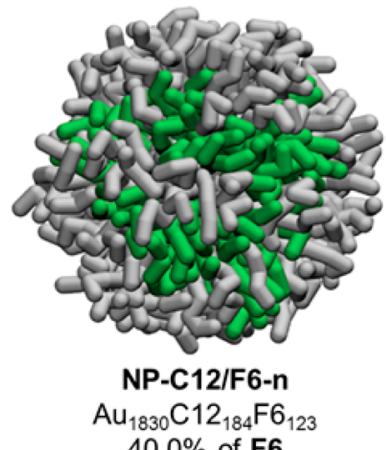

$40.0 \%$ of $\mathbf{F 6}$

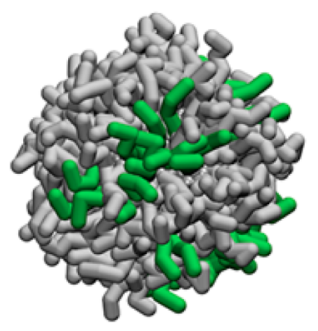

NP-C12/F6-g

$\mathrm{Au}_{1289} \mathrm{C} 12_{185} \mathrm{~F}_{46}$ $20.0 \%$ of $\mathbf{F 6}$

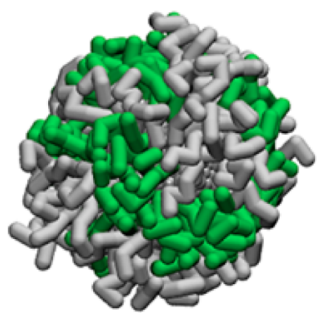

NP-C12/F6-o

$\mathrm{Au}_{1289} \mathrm{C}_{12}{ }_{123} \mathrm{~F}_{112}$ $47.8 \%$ of $\mathbf{F} 6$
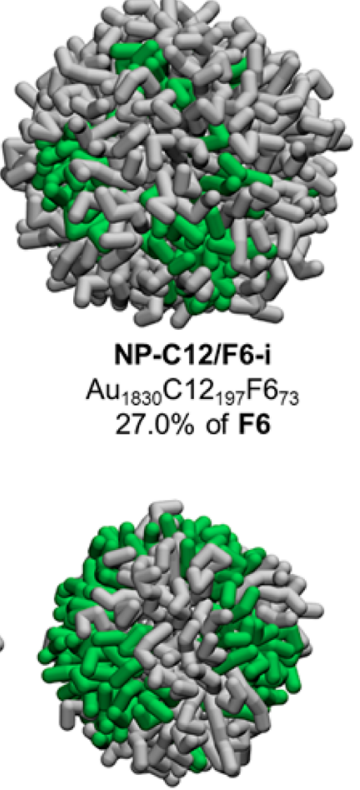

NP-C12/F6-p

$\mathrm{Au}_{976} \mathrm{C} 12{ }_{74} \mathrm{~F} 6{ }_{108}$ $60.0 \%$ of $\mathbf{F 6}$

Figure 11. Equilibrium morphologies of selected NP-C12/F6 (color code: gray, C12; green, F6) NPs as obtained by mesoscale simulations. Solvent is omitted for clarity.

At variance with NP-C12/F10, the alternative system featuring ligands of comparable lengths (i.e., NP-C8/F6) is characterized by a different shape of the variation of the chemical shift versus the percentage of F6 in the monolayer. In this case, an intermediate situation between $1 /(\mathrm{F} 6 \%)$ and a linear decay is observed (Figure 6). This trend is somewhat unexpected because, intuitively, the monolayers of NP-C8/F6 and the previously discussed NP-C12/F10 should both present a similar Janus-like organization. ${ }^{16}$

Yet, the corresponding mesoscopic simulations (see Figure 7) indisputably predict that the $\mathbf{C 8}$ and $\mathbf{F} 6$ thiolates, though similar in length, do not phase-separate in two distinct domains on the surface of the gold core. On the contrary, at small percentages of the fluorinated ligand (i.e., below 20\%), the F-thiolates prefer to remain isolated, yielding an essentially random organization. By increasing the loading, the fluorinated ligands do not form extended domains but rather tend to cluster in small, irregular patches. Overall, the morphology of NP-C8/F6 nanoparticles remains ill-defined, as no decisive evidence or reason for a preferred organization can be provided. While deeper and specific investigations on these aspects are underway, a working hypothesis can be anticipated: the shorter chain length of F6 with respect to F10 reduces the overall strength of the fluorophilic interactions, making the formation of fluorinated domains less favorable. In addition, the difference in steric bulk between $\mathrm{H}$ and F-thiolates may represent a bias in the organization of the thiolates when this geometrical mismatch is not counterbalanced by the occurrence of strong fluorophilic interactions.

Nanoparticles Coated by Ligands of Different Length, NP-C16/F6 and NP-C12/F6. The chemical shifts of the $\mathrm{CF}_{3}$ and $7-\mathrm{CF}_{2}$ groups as a function of the percentage of the F-ligand into the monolayer for the NP-C16/F6 system is shown in Figure 8.

The chemical shift versus composition curve is composed of three regions: an initial, almost linear decay is obtained in the composition range of $0-40 \%$, suggesting that by increasing the 
amount of fluorinated ligands into the monolayer these species experience very different chemical surroundings. In the region between 40 and $80 \%$, the chemical shift of the $\mathrm{CF}_{3}$ group is less sensitive to the composition of the monolayer, indicating that thiolate F6 experiences very similar chemical environments, even though the percentage of the fluorinated ligand into the monolayer increases. Lastly, when more than $80 \%$ of the $\mathbf{F 6}$ thiolate is introduced in the monolayer, the chemical shift of the terminal $\mathrm{CF}_{3}$ and of $7-\mathrm{CF}_{2}$ groups becomes again sensitive to the influence of the neighboring thiolates.

Mesoscale simulations on selected samples of NP-C16/F6 (Figure 9) revealed that the two ligands organize in striped domains on the surface of the gold NP core, even when $20 \%$ of the F6 thiolate is present in the monolayer. For these NPs, the gain in entropy due to the difference in length and the immiscibility of $\mathrm{H}$ - and F-ligands is sufficient to overcome the loss of van der Waals interactions between the hydrogenated alkyl chains, thus leading to a striped structure, in line with previously reported data about the organization of mixed monolayers composed of thiols having different length. ${ }^{16,33}$ Furthermore, the striped organization can explain why some samples of NP-C16/F6 are soluble in chloroform even at percentages of the fluorinated ligands into the monolayer up to $73 \%$. We reasoned that the sufficiently long hydrogenated chain might mask the short fluorinated ones, screening out the internanoparticle interactions between fluorinated domains if the width of the stripes is small enough, $0.75 \mathrm{~nm}$ on average. Evidence from simulations gives further insights into the monolayer organization as a function of the fluorinated thiolate percentage. At values of F6 less than 40\%, increasing the number of fluorinated ligands results in striped domains of F-chains different in size (and, consequently, in interface area), in line with the observed linear decay of the chemical shift. Once the stripes are completely formed (approximately at F6 > 40\%), further addition of fluorinated ligands does not influence the number of thiolates at the $\mathrm{H} / \mathrm{F}$ interface, as the incoming thiols locate inside a previously formed fluorinated stripe. At percentages of the fluorinated thiolate greater than $80 \%$, F6 chains prevail, thus reducing the size of the $\mathrm{H}$-domains and modifying the number of thiolates at the interface, ultimately resulting in a substantial decrease of the chemical shift of the diagnostic groups.

For the alternative system characterized by $\mathrm{F}$ - and $\mathrm{H}$-thiolates of different length, NP-C12/F6, the chemical shift of ${ }^{19} \mathrm{~F}$ nuclei in $\mathrm{CF}_{3}$ and $7-\mathrm{CF}_{2}$ as a function of the percentage of the fluorinated ligand is represented by a sigmoidal curve (Figure $10)$, which is very different from that obtained for the related NPC16/F6 system. In this case, a plateau region is found until $25 \%$ of fluorinated thiolate is introduced in the monolayer; this trend is followed by a sudden decrease of the chemical shift for small additions of F6 into the monolayer. Finally, a second plateau region is observed up to $100 \%$ F6. This can be an indication that, at low and high loadings of F6, there are no marked differences in the surroundings of the fluorinated ligands at the interface triggered by small variations of the monolayer composition. A sensible explanation for this behavior is that, within this interval of compositions, F-/H-regions of comparable size are gradually formed, and this does not influence the overall ratio between thiolates at the $\mathrm{H} / \mathrm{F}$ interface.

Mesoscale simulations reveal that the monolayer of nanoparticles NP-C12/F6 does not show a well-defined, ordered organization as a function of the F6 content. Indeed, as clearly shown in Figure 11, in some instances, stripe-like domains are formed, while at the same time, irregular patches are also observed. Specifically, when $10-15 \%$ of the fluorinated ligands are introduced into the monolayer, they form small patches typically composed of a few fluorinated chains. When the loading of F6 exceeds 30\%, elongated patches or stripe-like domains appear, and the dimensions of the relevant domains increase to $0.75-0.80 \mathrm{~nm}$, on average. This is also the composition at which the chemical shift of the $\mathrm{CF}_{3}$ and $7-\mathrm{CF}_{2}$ groups of $\mathbf{F} 6$ starts decreasing, indicating the generation of different environments around the fluorinated ligands. At percentages greater than $60 \%$ of F-thiolates, the shape of the domains is again predominantly patchy, and the chemical shift of the $\mathrm{CF}_{3}$ group becomes insensitive to any further variation of composition, suggesting no important changes in the neighboring environment. The predicted morphologies are also consistent with the solubility properties of NP-C12/F6. Indeed, with up to $40 \%$ of F6 in the monolayer, these systems are soluble in chloroform, indicating that the surfaces of the fluorinated domains are small enough to be efficiently shielded by the hydrogenated adjacent chains. Increasing the F6 content leads to an enlargement of the fluorinated domains, and the H-ligands do not hamper interparticle interactions anymore, leading to a change of the solubility properties.

HOESY Experiments. NOESY experiments on mixedmonolayer gold nanoparticles enable observation of crosspeaks between the spin systems of two different ligands if their distance is smaller than $0.4 \mathrm{~nm}$ and the number of spin systems involved is large enough. These conditions are fulfilled for random and striped mixed monolayers. ${ }^{22}$ Similar $2 \mathrm{D}$ NMR experiments between $\mathrm{F}$ - and $\mathrm{H}$-thiolates, that is, ${ }^{19} \mathrm{~F}-{ }^{1} \mathrm{H}$ HOESY, have been carried out on selected samples of each type of nanoparticle in order to support the morphological analysis described above. In particular, on the basis of the morphologies suggested by $1 \mathrm{D}$ NMR experiments and mesoscale simulations, cross-peaks are expected between the $\mathrm{CF}_{2}$ central groups of the fluorinated ligands and the methylene groups of the hydrogenated ones in the HOESY of nanoparticles NP-brC12/ F6, NP-C16/F6, NP-C12/F6, and NP-C8/F6. Indeed, as reported in Figure S7, the ${ }^{19} \mathrm{~F}-{ }^{1} \mathrm{H}$ HOESY spectra of these nanoparticles with a ratio between $\mathrm{F}$ - and $\mathrm{H}$-thiolates close to $1: 1$ display the presence of cross-peaks between the signal at about $-123 \mathrm{ppm}$, which is assigned to the central $\mathrm{CF}_{2}$ groups of the $\mathrm{F}$ ligands, and the peak at $1.2 \mathrm{ppm}$ of the methylene groups of the hydrogenated ones. In the HOESY spectra of nanoparticles NPbrC12/F6 and NP-C16/F6, a second cross-peak between the same $\mathrm{CF}_{2}$ central groups and a signal at around $2.5 \mathrm{ppm}$ in the ${ }^{1} \mathrm{H}$ NMR spectra could also be observed. This cross-peak was assigned to the intramolecular interaction between the $\mathrm{CH}_{2}$ and $\mathrm{CF}_{2}$ groups of the same thiolate $\mathbf{F} 6$ as from HOESY experiments on homoligand nanoparticles, NP-F6, and on the free thiol HF6.

Unexpectedly, the HOESY spectrum of NP-C12/F10-f presents a cross-peak between the signal of the central $\mathrm{CF}_{2}$ groups of the fluorinated ligands and the methylene groups of the hydrogenated ones. Since for Janus nanoparticles the fluorinated and hydrogenated thiolates are interacting solely at the interface between two domains, the intensity of this cross-peak is expected to be vanishingly small. An explanation for the observation of this cross-peak comes from the length of the fluorinated ligand; thiolate $\mathbf{F} 10$ has nine $\mathrm{CF}_{2}$ groups capable of determining a high number of interactions responsible for the NMR signal. For this reason, even though the $\mathrm{F}$-/ $\mathrm{H}$-ligands are interacting exclusively at the interface between the two phase-separated regions, the high number of $\mathrm{CF}_{2}$ groups may be sufficient to determine the appearance of the cross-peak. In order to test this hypothesis, we 
performed another HOESY analysis on a sample of the same class of nanoparticles presenting a low percentage (13.9\%) of the fluorinated thiolate into the monolayer (i.e., the NP-C12/F10-a system). For this system, the fluorinated ligands form a small patch (Figure 5), and accordingly, the number of $\mathrm{F} / \mathrm{H}$ interactions is reasonably lower with respect to NP-C12/F10f. Yet, even in this case, the HOESY spectrum exhibits a relatively intense cross-peak between the methylene groups of $\mathbf{C 1 2}$ and the central $\mathrm{CF}_{2}$ groups of F10. From these experiments, we conclude that the ${ }^{19} \mathrm{~F}-{ }^{1} \mathrm{H}$ HOESY is very sensitive and cross-peaks can be observed even though an exiguous number of interactions between the hydrogenated and fluorinated thiolates is present in the monolayer.

\section{CONCLUSIONS}

Our analysis of five different set of mixed-monolayer nanoparticles obtained by using blends of hydrogenated and fluorinated ligands and a plethora of different samples for each set nicely covering the loading of the F-ligand over the percentage range offers the basis to develop our understanding of the design rules required to control the formation of specifically patterned monolayer-protected NPs. Specifically, the combination of ${ }^{19} \mathrm{~F}$ NMR experiments and mesoscale simulation predictions allows one to assess the morphology of self-organized mixed monolayers comprising mixtures of $\mathrm{H}$ - and F-thiolates. Overall, the results point out that, besides the strong immiscibility between $\mathrm{H}$ - and F-ligands, other key parameterssuch as the high steric hindrance and rigidity of the fluorinated chains-influence the morphology of the organic layer. As a consequence, only when the mismatch of ligand length is equal to eight carbon atoms, stripe-like domains are formed (e.g., the NPC16/F6 system), driven by entropy gain. On the other hand, a length difference of four carbon atoms gives rise to domains that appear as patches or elongated patches, as seen for the NP-C12/ F6 system. Ligands of the same length self-organize in Janus domains (NP-C12/F10), as expected. However, if both ligands are shorter, as in NP-C8/F6, the experimental and theoretical results suggest the formation of a SAM with no specific morphology. Finally, the presence of a branched ligand in the monolayer, such as in the NP-brC12/F6 system, disfavors phase segregation and promotes a random organization of the two types of ligands. The design principles arising from our analysis, although obtained with simple model systems, are envisaged to be of general validity and, as such, are currently being employed by our group in the synthesis and characterization of more complex nanoparticle systems for practical applications.

\section{METHODS}

Synthesis. The detailed synthetic procedures for the preparation of gold nanoparticles NP-C16/F6, NP-C12/F6, NP-C12/F10, NP-C8/ F6, and NP-brC12/F6 are reported in ref 29. Synthesis of NP-C8/F6-i and NP-C8/F6-p systems is given in full in the SI.

NMR Experiments. NMR experiments were carried out on a Varian 500 spectrometer (operating at $500 \mathrm{MHz}$ for ${ }^{1} \mathrm{H}$ and at $470.08 \mathrm{MHz}$ for $\left.{ }^{19} \mathrm{~F}\right)$. The ${ }^{1} \mathrm{H}$ chemical shifts are referenced to the residual protons in deuterated solvents, whereas $\mathrm{CFCl}_{3}$ was used as an external reference for

${ }^{19} \mathrm{~F}$ spectra. The maximum error on ${ }^{19} \mathrm{~F}$ chemical shift of repeated measurements is $0.04 \mathrm{ppm}$.

Samples were prepared by dissolving about $10 \mathrm{mg}$ of NPs in $0.7 \mathrm{~mL}$ of deoxygenated $\mathrm{CDCl}_{3}$ or mixtures of $\mathrm{CDCl}_{3} / \mathrm{C}_{6} \mathrm{~F}_{6}$, as detailed in Tables S1-S5 of the SI, and additionally deoxygenated by bubbling argon while keeping the NMR tube in an ultrasound bath for at least $1 \mathrm{~min}$. For ${ }^{19} \mathrm{~F}-{ }^{1} \mathrm{H}$ HOESY experiments, the pulse sequence $\mathrm{FH}$ _HOESY was used with 256 scans and a tuning increment, $t_{1}$, of 128 .
Computational Studies. Coarse-grained methods as DPD are especially suitable for predicting self-assembling phenomena as involved in the organization of binary mixtures of ligands on solid curved surfaces because they allow longer sampling times with respect to atomic resolution techniques (e.g., $\mathrm{MD})$. The computational approach applied here consists of exploiting information obtained at lower-level simulations (e.g., MD simulations) as input for higher-level calculations (e.g., mesoscopic DPD simulations). In detail, a suitable atomistic model of the gold/ligands/solvent interface was employed to estimate the interaction energy among the different system components. These energy values were, in turn, utilized to derive the corresponding DPD simulation parameters. Further, by matching the atomistic/mesoscale pair-pair correlation functions for each ligand, the coarse-grained topology of each chain ligand was calculated. The computational procedure employed here was already successfully applied by our group $^{28,34-38}$ to predict the self-assembling organization of several immiscible ligand mixtures, including poly(ethylene oxide)-terminating hydrocarbon/perfluorocarbon thiolated chains as well as mercaptoundecanesulfonate/octanethiol ligands on a gold nanoparticle spherical surface and other immiscible polymer-related systems.

\section{ASSOCIATED CONTENT}

\section{S Supporting Information}

The Supporting Information is available free of charge on the ACS Publications website at DOI: 10.1021/acsnano.6b03931.

${ }^{19}$ F NMR of the thiol HF6 and of NP-C12/F6 with peak assignment; description of the experiments to determine the influence of solvent composition on the chemical shift of the NP-C16/F6; preparation of nanoparticles NP-C8/ F6-i and NP-C8/F6-p; Tables S1-S5 reporting the characterization data of all nanoparticles, ${ }^{19} \mathrm{~F}$ chemical shifts of $\mathrm{CF}_{3}$ and 7 - or 9- $\mathrm{CF}_{2}$ groups, and percentage of $\mathrm{C}_{6} \mathrm{~F}_{6}$ added; ${ }^{19} \mathrm{~F}-{ }^{1} \mathrm{H}$ HOESY experiments; details of the computational methods, mesoscale models, and mesoscale procedure (PDF)

\section{AUTHOR INFORMATION}

\section{Corresponding Authors}

*E-mail: paola.posocco@dia.units.it.

*E-mail: lpasquato@units.it.

\section{Author Contributions}

${ }^{\perp}$ P. Posocco and L. Pasquato are senior co-authors.

Notes

The authors declare no competing financial interest.

\section{ACKNOWLEDGMENTS}

L. Pasquato and P. Pengo dedicate this manuscript to the memory of Professor V. Lucchini, who started the NMR school at the University of Padova and passed away March 22, 2016. The financial support of projects FIRB prot. RBAP11ETKA, MIUR: Project MULTINANOITA, and University of Trieste (FRA projects 2014 and 2015) is gratefully acknowledged. This work is generously supported by the Italian Ministry of University Research (MIUR) through the Scientific Independence of Young Researchers (SIR) project "Structure and Function at the Nanoparticle biointerface” (Grant RBSI14PBC6 to P.Posocco). The authors are grateful to Prof. Chiara Schmid for her support with the thermogravimetric analyses.

\section{REFERENCES}

(1) Oeggerli, M. http://ngm.nationalgeographic.com/2009/12/ pollen/oeggerli-photography (last accessed September 19, 2016).

(2) Kalia, M.; Jameel, S. Virus Entry Paradigms. Amino Acids 2011, 41, 1147-1157. 
(3) Whiteford, J. R.; Spanu, P. D. Hydrophobins and the Interactions Between Fungi and Plants. Mol. Plant Pathol. 2002, 3, 391-400.

(4) Zhang, Z.; Horsch, M. A.; Lamm, M. H.; Glotzer, S. C. Tethered Nano Building Blocks: Toward a Conceptual Framework for Nanoparticle Self-Assembly. Nano Lett. 2003, 3, 1341-1346.

(5) Du, J.; O' Reilly, R. K. Anisotropic Particles with Patchy, Multicompartment and Janus Architectures: Preparation and Application. Chem. Soc. Rev. 2011, 40, 2402-2416.

(6) Walther, A.; Müller, A. H. E. Janus Particles: Synthesis, SelfAssembly, Physical Properties, and Applications. Chem. Rev. 2013, 113, 5194-5261.

(7) Bianchi, E.; Kahl, G.; Likos, C. N.; Sciortino, F. Patchy Particles. J. Phys.: Condens. Matter 2015, 27, 230301.

(8) Zhou, Y.; Marson, R. L.; van Anders, G.; Zhu, J.; Ma, G.; Ercius, P.; Sun, K.; Yeom, B.; Glotzer, S. C.; Kotov, N. A. Biomimetic Hierarchical Assembly of Helical Supraparticles from Chiral Nanoparticles. ACS Nano 2016, 10, 3248-3256.

(9) Liu, X.; Wu, F.; Tian, Y.; Wu, M.; Zhou, Q.; Jiang, S.; Niu, Z. Size Dependent Cellular Uptake of Rod-Like Bionanoparticles with Different Aspect Ratios. Sci. Rep. 2016, 6, 24567.

(10) Dag, A.; Zhao, J.; Stenzel, M. H. Origami with ABC Triblock Terpolymers Based on Glycopolymers: Creation of Virus-Like Morphologies. ACS Macro Lett. 2015, 4, 579-583.

(11) Huang, R.; Carney, R. P.; Ikuma, K.; Stellacci, F.; Lau, B. L. T. Effects of Surface Compositional and Structural Heterogeneity on Nanoparticle-Protein Interactions: Different Protein Configurations. ACS Nano 2014, 8, 5402-5412.

(12) Li, Z.-W.; Zhu, Y.-L.; Lu, Z.-Y.; Sun, Z.-Y. A Versatile Model for Soft Patchy Particles with Various Patch Arrangements. Soft Matter 2016, 12, 741-749.

(13) Gentilini, C.; Pasquato, L. Morphology of Mixed-Monolayers Protecting Metal Nanoparticles. J. Mater. Chem. 2010, 20, 1403-1412.

(14) Jackson, A. M.; Myerson, J. W.; Stellacci, F. Spontaneous Assembly of Subnanometre-Ordered Domains in the Ligand Shell of Monolayer-Protected Nanoparticles. Nat. Mater. 2004, 3, 330-336.

(15) Jackson, A. M.; Hu, Y.; Silva, P. J.; Stellacci, F. From Homoligandto Mixed-Ligand- Monolayer-Protected Metal Nanoparticles: A Scanning Tunneling Microscopy Investigation. J. Am. Chem. Soc. 2006, 128, 11135-11149.

(16) Singh, C.; Ghorai, P. K.; Horsch, M. A.; Jackson, A. M.; Larson, R. G.; Stellacci, F.; Glotzer, S. C. Entropy-Mediated Patterning of Surfactant-Coated Nanoparticles and Surfaces. Phys. Rev. Lett. 2007, 99, 226106.

(17) Carney, R. P.; DeVries, G. A.; Dubois, C.; Kim, H.; Kim, J. Y.; Singh, C.; Ghorai, P. K.; Tracy, J. B.; Stiles, R. L.; Murray, R. W.; Glotzer, S. C.; Stellacci, F. Size Limitations for the Formation of Ordered Striped Nanoparticles. J. Am. Chem. Soc. 2008, 130, 798-799.

(18) Verma, A.; Uzun, O.; Hu, Y.; Hu, Y.; Han, H.-S.; Watson, N.; Chen, S.; Irvine, D. J.; Stellacci, F. Surface-Structure-Regulated CellMembrane Penetration by Monolayer-Protected Nanoparticles. Nat. Mater. 2008, 7, 588-595.

(19) Stewart, A.; Zheng, S.; McCourt, M. R.; Bell, S. E. J. Controlling Assembly of Mixed Thiol Monolayers on Silver Nanoparticles to Tune their Surface Properties. ACS Nano 2012, 6, 3718-3726.

(20) Harkness, K. M.; Balinski, A.; Mclean, J. A.; Cliffel, D. E. Nanoscale Phase Segregation of Mixed Thiolates on Gold Nanoparticles. Angew. Chem., Int. Ed. 2011, 50, 10554-10559.

(21) Guarino, G.; Rastrelli, F.; Scrimin, P.; Mancin, F. LanthanideBased NMR: A Tool to Investigate Component Distribution in MixedMonolayer-Protected Nanoparticles. J. Am. Chem. Soc. 2012, 134, $7200-7203$.

(22) Liu, X.; Yu, M.; Kim, H.; Mameli, M.; Stellacci, F. Determination of Monolayer-Protected Gold Nanoparticle Ligand-Shell Morphology Using NMR. Nat. Commun. 2012, 3, 1182.

(23) Tsao, M.-W.; Rabolt, J. F.; Schönherr, H.; Castner, D. G. Semifluorinated/Hydrogenated Alkylthiol Thin Films: A Comparison between Disulfides and Thiol Binary Mixtures. Langmuir 2000, 16, 1734-1743.
(24) Dass, A.; Guo, R.; Tracy, J. B.; Balasubramanian, R.; Douglas, A. D.; Murray, R. W. Gold Nanoparticles with Perfluorothiolate Ligands. Langmuir 2008, 24, 310-315.

(25) Im, J.; Chandekar, A.; Whitten, J. E. Anomalous Vapor Sensor Response of a Fluorinated Alkylthiol-Protected Gold Nanoparticle Film. Langmuir 2009, 25, 4288-4292.

(26) Weinberg, D. J.; He, C.; Weiss, E. A. Control of the Redox Activity of Quantum Dots Through Introduction of Fluoroalkanethiolates into their Ligand Shells. J. Am. Chem. Soc. 2016, 138, 2319-2326.

(27) Gentilini, C.; Franchi, P.; Mileo, E.; Polizzi, S.; Lucarini, M.; Pasquato, L. Formation of Patches on 3D SAMs Driven by Thiols with Immiscible Chains Observed by ESR Spectroscopy. Angew. Chem., Int. Ed. 2009, 48, 3060-3064.

(28) Posocco, P.; Gentilini, C.; Bidoggia, S.; Pace, A.; Franchi, P.; Lucarini, M.; Fermeglia, M.; Pricl, S.; Pasquato, L. Self-Organization of Mixtures of Fluorocarbon- and Hydrocarbon Amphiphilic Thiolates on the Surface of Gold Nanoparticles. ACS Nano 2012, 6, 7243-7253.

(29) Şologan, M.; Cantarutti, C.; Bidoggia, S.; Polizzi, S.; Pengo, P.; Pasquato, L. Routes to the Preparation of Mixed Monolayers of Fluorinated and Hydrogenated Alkanetiolates Grafted on the Surface of Gold Nanoparticles. Faraday Discuss. 2016, DOI: 10.1039/ C6FD00016A.

(30) Kohlmann, O.; Steinmetz, W. E.; Mao, X.-A.; Wuelfing, W. P.; Templeton, A. C.; Murray, R. W.; Johnson, C. S., Jr. NMR Diffusion, Relaxation, and Spectroscopic Studies of Water Soluble MonolayerProtected Gold Nanoclusters. J. Phys. Chem. B 2001, 105, 8801-8809 and references cited therein.

(31) Pradhan, S.; Brown, L. E.; Konopelski, J. P.; Chen, S. Janus Nanoparticles: Reaction Dynamics and NOESY Characterization. J. Nanopart. Res. 2009, 11, 1895-1903.

(32) Shimizu, T.; Teranishi, T.; Hasegawa, S.; Miyake, M. Size Evolution of Alkanethiol-Protected Gold Nanoparticles by Heat Treatment in the Solid State. J. Phys. Chem. B 2003, 107, 2719-2724.

(33) Ghorai, P. K.; Glotzer, S. C. Atomistic Simulation Study of Striped Phase Separation in Mixed-Ligand Self-Assembled Monolayer Coated Nanoparticles. J. Phys. Chem. C 2010, 114, 19182-19187.

(34) Posocco, P.; Hassan, Y. M.; Barandiaran, I.; Kortaberria, G.; Pricl, S.; Fermeglia, M. Combined Mesoscale/Experimental Study of Selective Placement of Magnetic Nanoparticles in Diblock Copolymer Films Via Solvent Vapor Annealing. J. Phys. Chem. C 2016, 120, 7403-7411.

(35) Posel, Z.; Posocco, P.; Lisal, M.; Fermeglia, M.; Pricl, S. Highly Grafted Polystyrene/Polyvinylpyridine Polymer Gold Nanoparticles in a Good Solvent: Effects of Chain Length and Composition. Soft Matter 2016, 12, 3600-3611.

(36) Posel, Z.; Posocco, P.; Fermeglia, M.; Lísal, M.; Pricl, S. Modeling Hierarchically Structured Nanoparticle/Diblock Copolymer Systems. Soft Matter 2013, 9, 2936-2946.

(37) Posocco, P.; Posel, Z.; Fermeglia, M.; Lísal, M.; Pricl, S. A Molecular Simulation Approach to the Prediction of the Morphology of Self-Assembled Nanoparticles in Diblock Copolymers. J. Mater. Chem. 2010, 20, 10511-10520.

(38) Scocchi, G.; Posocco, P.; Handgraaf, J.-W.; Fraaije, J. G. E. M.; Fermeglia, M.; Pricl, S. A Complete Multiscale Modelling Approach for Polymer-Clay Nanocomposites. Chem. - Eur. J. 2009, 15, 7586-7592. 